\title{
Inteligencia emocional y percepción de las emociones básicas como un probable factor contribuyente al mejoramiento del rendimiento en las ventas: Una investigación teórica*
}

\author{
Emotional Intelligence and Perception of Basic \\ Emotions as a Possible Contributing Factor to Improve \\ Sales Performance: A Theoretical Research
}

Recibido: 16 de junio de 2014| Aceptado: 09 de diciembre de 2014

\author{
Jean David Polo-Vargas ** \\ MILTON ZAMBRANO *** \\ Fundación Universidad del Norte, Colombia \\ ANDRÉS MUÑOZ ***** \\ Fundación Universidad del Norte, Colombia \\ Estudios y Datos. S.A.S, Colombia \\ JORGE VELILLA ****** \\ Fundación Universidad del Norte, Colombia
}

doi: 10.11144/Javeriana.upsy15-2.iepe

Para citar este artículo: Polo-Vargas, J.D., Zambrano, M., Muñoz, A., \& Velilla, J. (2016). Inteligencia emocional y percepción de las emociones básicas como un probable factor contribuyente al mejoramiento del rendimiento en las ventas: Una investigación teórica. Universitas Psychologica, 15(2), 73-86. http://dx.doi.org/10.11144/Javeriana. upsy15-2.iepe

Artículo de investigación.

** Docente investigador. Becario de la fundación COLFUTURO Y COLCIENCIAS promoción 2008. Correo electrónico: pjean@uninorte.edu.co

**** Asistente de investigación del grupo de investigación en psicología en la línea organización y gestión del talento. Correo electrónico: curciom@uninorte.edu.co

****** Docente e Investigador en Estudios de Mercados y Comportamiento del Consumidor. Correo electrónico: aalvis@uninorte.edu.co

${ }^{* * * * * * * *}$ Asistente de investigación del grupo de investigación en psicología en la línea organización y gestión del talento humano. Correo electrónico: jguardela@ uninorte.edu.co

\section{RESUMEN}

La inteligencia emocional es uno de los conceptos que ha empezado a transformar el mundo empresarial gracias a los avances en el reconocimiento y el manejo de las emociones. Este es un campo que permitirá la elaboración de estrategias para robustecer los procesos de desarrollo organizacional. El presente artículo de investigación bibliográfica, sistematiza y aporta a la producción científica sobre el concepto de inteligencia emocional y sus potenciales aplicaciones al proceso de ventas. El objetivo de este estudio fue el de conceptualizar acerca del rol que puede jugar la percepción de los distintos estados emocionales en el proceso de ventas y cómo esto puede ayudar a mejorar el rendimiento empresarial.

Palabras clave

inteligencia emocional; percepción emocional; proceso de ventas; rendimiento

\section{A B S T R A C T}

Emotional intelligence is one of the concepts that have begun to transform the business world thanks to the advancements in emotional management and recognition. This is a field that allows the elaboration of strategies for the strengthening of organizational development processes. The present article of bibliographic research incorporates part of the scientific production about the concept of emotional intelligence and the possible application of this concept into the sales process. The objective is to conceptualize about the role that the perception of different emotional states can play in the sales process and how it can help improve organizational performance. Keywords emotional intelligence; emotional perception; sale process; performance 


\section{Introducción}

La interacción entre comprador y vendedor es importante y de ser eficaz puede incrementar la satisfacción y retención del primero (Ahearne, Jelinek, $\&$ Jones 2007). Marina (1993), citada por Rodríguez y Suárez (2012), afirma que si bien las ciencias cognitivas han hecho valiosos aportes al estudio de la inteligencia humana, estos no se deben enfocar solo en la racionalidad sino también en las emociones y aproximarse a una visión holística de la persona. La inteligencia emocional permite abordar la dimensión afectiva de la naturaleza humana y, al mismo tiempo, obtener una mejor y más amplia explicación acerca de por qué la inteligencia general (cociente intelectual) no predice tanto el éxito personal y/o el social (Rodríguez, Amaya \& Argota, 2011).

Aun así, según Bagozzi, Gopinath y Nyer (1999), el reconocimiento de los diversos estados emocionales por medio de la expresión facial es un área comúnmente descuidada por los vendedores. Las emociones constituyen una fuerza psicológica que puede influenciar el comportamiento y el rendimiento de los profesionales en ventas (Brown, Cron, \& Slocum, 1997). En consecuencia, es imperativo tener en cuenta los diversos estados emocionales y, como nos plantean Clopton, Stoddard y Clay (2002), poseer una buena disposición para optimizar las percepciones que tienen los compradores de sus vendedores para aumentar posibles negocios en el futuro.

Se puede afirmar que los estados emocionales afectan la dirección del pensamiento cuando somos capaces de reconocer altos estados de ánimo en otros individuos, facilitándose el cambio de actitud en estas mismas personas (Briñol, Gandarillas, Horcajo, \& Becerra, 2010). Por este motivo, es importante que los vendedores ejerzan un grupo de habilidades mentales que consistan en la percepción, utilización, entendimiento y manejo de los diversos estados emocionales, todo esto comprendido dentro de la teoría de la inteligencia emocional elaborada por Salovey y Grewal (2005).

Dentro de este grupo de habilidades el carácter fundamental consiste en la percepción de los estados emocionales que, como nos afirma Ekman
(2003), manifiestan los cuerpos de maneras diferentes por medio de indicadores musculares específicos y distintos para cada tipo de emoción básica, esto se denomina expresión facial. Es importante que el vendedor pueda distinguir entre estos estados emocionales primordiales para así mejorar su efectividad. En este estudio se considera la interacción económica como un proceso social con etapas definidas y, al mismo tiempo, con formas específicas de comportamiento esperado entre las partes que desarrollan esta interacción (Biggart \& Delbridge, 2004). Esta perspectiva es un cambio de postura de un menor precio a una relación a largo plazo entre vendedor y comprador en donde se presenta el beneficio mutuo (Plouffe, Holmes, \& Beuk, 2013).

Una vez que el estado emocional apropiado es identificado, se vuelve de suma importancia que el vendedor presente una orientación hacia el cliente a la hora de realizar el proceso de ventas, así se establecerá una relación con base en lo que el cliente necesita comprar sin necesidad de convencerlo de cosas que no podría necesitar (Saxe \& Weitz, 1982). También es perentorio que el vendedor ajuste su comportamiento a las demandas de la interacción de ventas. Todo esto conlleva a resultados asociados con una venta positiva, los cuales consisten en la percepción obtenida del cliente acerca de la transacción pudiendo llegar a afectar la probabilidad de futuras ventas (Plouffe et al., 2013).

En concordancia con lo anterior, el objetivo que se buscó en este estudio fue el de identificar y analizar los conceptos fundamentales que se asocian con la inteligencia emocional, la percepción emocional, el proceso de ventas y rendimiento, para la construcción de un marco de referencia que sirva para la elaboración de un software de entrenamiento en ventas. Por tanto, este primer ejercicio conceptual nos permitirá mejorar la calidad de la atención de los vendedores además de su competitividad.

\section{Método}

La investigación apunta a una revisión sistemática de la evidencia científica. Esto consiste en unos estudios secundarios que, según Perestelo-Pérez (2013), es utilizado para identificar, seleccionar, evaluar, analizar 
y sintetizar los estudios empíricos que permitirán responder a problemáticas específicas. Se tomaron fuentes primarias, secundarias y terciarias sobre los temas a abordar: inteligencia emocional, percepción emocional, proceso de ventas y rendimiento. Una vez seleccionadas las bases de datos (ISI Web Of Knowledge, EBSCO, ProQuest y Scopus), se procedió a seleccionar los artículos y, luego, a elaborar las categorías de análisis las cuales emergieron desde la teoría consultada; la revisión conceptual se realizó desde agosto de 2013 hasta mayo de 2014.

\section{Resultados}

\section{La inteligencia emocional}

El concepto de inteligencia emocional surge debido a las investigaciones llevadas a cabo por John D. Mayer y Peter Salovey (1997) que, a su vez, se basan en los adelantos de Howard Gardner al introducir en su publicación de 1983 el concepto de las inteligencias múltiples. Esta construcción teórica sobre la importancia de las emociones ha sido trabajada por diversos autores tal como Cortés, Barragán y Vásquez (2002), quienes sostienen que la inteligencia emocional comprende tanto la evaluación como la expresión de las emociones propias y las de los demás, así como la comprensión de las experiencias emocionales para promover un crecimiento emocional e intelectual.

Los autores mencionados (Cortés, Barragán, \& Vásquez, 2002), sugieren diez factores constituyentes de la inteligencia emocional:

1. Control emocional: control de los sentimientos y manejo del humor.

2. Autoestima: motivación de sí mismo.

3. Control de los impulsos: control de los impulsos y aceptación de la gratificación tardía o posterior.

4. Manejo del estrés: su control y manejo ante el cambio.

5. Habilidad social: relación y empatía con otros.

6. Balance creativo: mantener el equilibrio entre el trabajo, los deberes del hogar y conservar el buen humor.
7. Habilidad de comunicación: comunicación efectiva con otras personas.

8. Manejo de metas: fijarse metas realistas en todas las áreas de la vida.

9. Auto motivación: auto motivación para alcanzar las metas de nuestra vida.

10. Actitudes positivas: mantenerse en actitud positiva y realista, aun en tiempos difíciles.

Por su parte Mathewas (2002) citado por Hassan y Mohsen (2012) define la inteligencia emocional como la forma en la que un individuo, lidia con la experiencia emocional y administra sus emociones mientras se comunica con otros; en otras palabras, la inteligencia emocional afecta cómo nos percibimos a nosotros mismos y la manera como nos comunicamos con los demás.

En este mismo sentido, autores como Petridges, Furnham y Mavroveli (2007) citados por Chopra y Kanji (2010), proponen una distinción conceptual entre el modelo basado en habilidades y el modelo basado en rasgos de la inteligencia emocional. Por su parte, el modelo basado en habilidades se fundamenta en destrezas que han probado ser resistentes a la medición científica, y en cuanto al modelo basado en rasgos, este se basa en disposiciones comportamentales y habilidades de auto-percepción, considerando la inteligencia emocional como un conjunto de rasgos de personalidad que pueden medirse por medio de instrumentos de auto-reporte.

Desde este último, Trujillo y Rivas (2008) afirman que la inteligencia emocional es una serie de habilidades adaptativas que se llevan a cabo gracias a criterios como la evaluación y la expresión de emociones, la regulación de emociones y la utilización de emociones de una manera adaptativa. Su escala es abreviada TMMS (trait meta-mood scale) y está constituida por 48 reactivos divididos en 24 ítems de emociones, 12 de claridades acerca de las emociones y 12 de reparación emocional.

Teniendo en cuenta las bases referenciales del estudio sobre la inteligencia emocional, Mayer y Salovey (1997) la consideran como un conjunto de habilidades mentales que se ocupan de las emociones y el procesamiento de la información emocional. Estas habilidades se organizan jerárquicamente 
desde las más simples hasta las más complejas, incluyendo: (1) la habilidad de percibir emociones, (2) la habilidad de utilizar emociones para facilitar el razonamiento, (3) la habilidad de entender el significado de las emociones y la información que transmiten; Y (4) la habilidad de efectivamente manejar y administrar las emociones:

1. Percibir emociones: es la habilidad fundamental de la inteligencia emocional, esta permite todo el procesamiento de la información emocional. Aquí se tiene en cuenta la aptitud para identificar correctamente cómo se sienten las personas.

2. Utilizar emociones: consiste en aprovechar y acceder a las emociones para facilitar varias actividades cognitivas, tales como pensar y resolver problemas, por ejemplo: un estado de ánimo triste o bajo puede posibilitar el trabajo metódico, que requiera atender a detalles y buscar errores. Por otro lado, un estado de ánimo alto, puede estimular el pensamiento creativo e innovador. En conclusión, es imperativa la aptitud para crear emociones y para integrar los sentimientos en la forma de pensar.

3. Entender o comprender emociones y el conocimiento emocional: es la habilidad de comprender el lenguaje emocional y, a la vez, apreciar relaciones complejas entre emociones, esto comprende la habilidad de ser sensitivo a las pequeñas variaciones entre emociones, además incluye la capacidad de reconocer y describir cómo las emociones, en medio de las relaciones interpersonales, evolucionan con el paso del tiempo para comprender sus causas.

4. Manejar, administrar y regular emociones: consiste en la habilidad de organizar emociones, tanto en otras personas como en nosotros mismos, para así conseguir un crecimiento emocional e intelectual. Esto podrá generar estrategias eficaces dirigidas a utilizar, de forma controlada, las emociones de forma que ayuden a la consecución de las metas propias, en lugar de verse influido por las propias emociones de forma imprevisible.

Según Extremera y Fernández-Berrocal (2004) la sub-escala de manejo emocional del MSCEIT tuvo un papel muy importante en la predicción de aspectos de funcionamiento social. Estos hallazgos demuestran que las habilidades de manejo emo- cional predicen mejores interacciones positivas y menores interacciones negativas.

\section{La personalidad, el desempeño y la inteligencia emocional}

En cuanto a la personalidad, Nawin, Redzuan y Hamsan (2012) la definen como un concepto abstracto que implica acciones, emociones, el reconocimiento y motivaciones de una persona, los seres humanos tienen una personalidad única y esta se mantiene constante a lo largo del tiempo. Los autores también tienen en cuenta la personalidad como un conjunto de rasgos psicológicos y mecanismos dentro del individuo que perduran y han influido en su interacción con la adaptación al medio ambiente.

En la actualidad, uno de los modelos más importantes sobre la personalidad es el denominado Big Five cuyo supuesto básico es que cinco factores, cada uno con polos opuestos, son suficientes para describir la personalidad; tales factores reciben diversas denominaciones dependiendo de cada autor. Siguiendo a Norman (1963), citado en A. García-Izquierdo, Ramos-Villagrasa y M. GarcíaIzquierdo (2009), el primero es el Neuroticismo $(\mathrm{N})$, que se refiere a la preocupación, inseguridad y la tensión; el segundo es la Extraversión (E), que indica sociabilidad, locuacidad y optimismo; el tercero es la Apertura a la experiencia $(\mathrm{O})$, originalidad, creatividad e independencia; el cuarto es la Amigabilidad (A), que consiste en compasión, confianza y cortesía; y por último, el quinto factor es la Conciencia $(\mathrm{C})$, que comprende la diligencia, la fiabilidad y la organización.

Asi mismo, Ather \& Bano (2013) definen la personalidad como la realización suprema de la conducta peculiar innata del ser humano, considerando los rasgos de personalidad al tener una fuerte relación con la inteligencia emocional. Desde el modelo de los cinco factores de rasgos, las medidas de la inteligencia emocional correlacionan significativamente con la extraversión y el neuroticismo, además de poseer una correlación positiva aunque más leve con la amabilidad, la apertura a la experiencia y la escrupulosidad (Petrides \& Furnham; Saklofske, Eustin, \& Minski, 
citados por Ather \& Bano, 2013). Otros autores (Brackett \& Mayer, 2003, citados en Ather \& Bano, 2013) encontraron que la inteligencia emocional se correlaciona muy significativamente con el neuroticismo, la extraversión, la agradabilidad y la conciencia, pero moderadamente en relación con la apertura a la experiencia.

Sin embargo, cuando se utilizó la prueba de Inteligencia Emocional Mayer-Salovey-Caruso (MSCEIT; Mayer, Salovey, \& Caruso, 2002) solo la apertura a la experiencia y la amabilidad se relacionó con la inteligencia emocional (Mayer, Salovey, \& Caruso, 2002). Asimismo, esta conexión entre la inteligencia emocional y los rasgos de personalidad es relevante para la comprensión y el control de las emociones de vital importancia para la construcción de la personalidad. La relación entre ambas variables ha sido ampliamente investigada, pero el nivel de relación entre estas dos construcciones depende de las medidas utilizadas para evaluar.

La relación entre la personalidad y el rendimiento en el trabajo siempre ha sido un tema de investigación importante desde la psicología (Ghiselli, 1973; Guion \& Gottier, 1965, citados por Sitser, van der Linden, \& Born, 2013), sin embargo, la personalidad ha tenido una reputación mixta como predictor de los resultados del trabajo. Campbell (1990) citado por Sitser, et al. (2013), sugirió una estrategia de alineación en la que son identificadas las características de personalidad que subyacen en tipos específicos de rendimiento en el trabajo. Por ejemplo, el rasgo de personalidad referente a la orientación al detalle - lo que refleja la tendencia de enfocar y comprobar los detalles a fondo- puede ser la base de rendimiento en tareas administrativas en las que es importante ser sistemático y trabajar a través de información detallada a fondo.

El estudio llevado a cabo por Sitser et al. (2013) examinó las vigencias de predicción de tres niveles de medidas de personalidad en dos niveles de los criterios de desempeño en el trabajo a partir de los cinco grandes factores de personalidad. Esto indicó que los empleados de ventas que son altruistas, emocionalmente estables, agradables, conscientes, extrovertidos e intelectualmente abiertos, presentan altos niveles de bienestar, satisfacción con la vida, autoestima e inteligencia emocional, estando en mejores condiciones de alcanzar nuevos clientes.

\section{El modelo de emociones básicas}

Es menester distinguir cual modelo teórico será abordado a la hora de tratar el tema de las emociones, aquí se distingue el modelo de Ekman (1994) quien propone que las emociones "básicas" son las emociones biológicas obtenidas mediante el proceso de evolución; Ekman (1992) utiliza la expresión facial para estudiar las emociones, esta expresión genera una fisiología única y una actividad cerebral asociada con las emociones, además tiene una trascendencia cultural, es decir, se presenta de manera general y distintiva. Las emociones espontáneas pueden ser distinguidas de las emociones fingidas en su expresión. Durante el transcurso de la investigación, se establecen en primera instancia una lista de emociones que son las primordiales: la ira, el miedo, la sorpresa, la tristeza, la felicidad, el asco y el desprecio (Ekman, 1992).

Posteriormente, Ekman (1999) genera un criterio para determinar cuáles son las emociones básicas y este criterio consiste en que dicha emoción presente las siguientes características:

- Señales distintivas y universales.

- Presencia en otros primates.

- Fisiología distintiva.

- Distinciones universales en eventos anteriores.

- Inicio rápido.

- Corta duración.

- Evaluación automática.

- Ocurrencia espontánea.

- Un desarrollo distintivo en apariencia.

- Memorias, pensamientos e imágenes distintivas.

- Una experiencia subjetiva distintiva.

Con este criterio Ekman (1999) establece el modelo de emociones básicas, las cuales están constituidas por la ira, el miedo la tristeza, el disfrute o goce, el asco y la sorpresa. Un aporte sugestivo para el estudio del manejo de emociones es el de las neurociencias, las cuales esbozaremos a continuación. 


\section{Las neurociencias}

Las neurociencias aplicadas al comportamiento del consumidor, o neuromarketing, tratan el estudio del funcionamiento del cerebro durante las decisiones de la compra de un producto y de las reacciones presentadas por los consumidores a la hora de efectuar estas decisiones (Salazar, 2011). Estás miden cómo el cerebro responde a determinados estímulos que provienen de la publicidad, no solo se tienen en cuenta estos procesos fisiológicos, sino también se evalúan los resultados de las acciones desarrolladas en torno a las ventas, la percepción de las marcas y las preferencias, por lo que este enfoque considera el proceso de consumo desde el inicio hasta su final incluyendo sus bases fisiológicas.

Asimismo, Vecchiato et al. (2011) mencionan varias técnicas utilizadas dentro del neuromarketing, como son la resonancia funcional magnética (RFM) y los estudios de los potenciales evocados; los cuales permiten una medida directa y una localización inmediata de la actividad cerebral. En consecuencia, estas técnicas permiten que las medidas puedan ser consideradas más objetivas y útiles que las medidas tradicionales, como los cuestionarios o las autoevaluaciones, instrumentos que resultan altamente costosos y de compleja aplicación.

A su vez, Ambler, Braeutigam, Stins, Rose y Swithenby (2004) afirman que los potenciales evocados hacen referencia a señales bioeléctricas, producto de la actividad de las zonas neurales, periférica y central como respuesta a estímulos externos. Los estudios de los potenciales evocados consisten en la aplicación de un estímulo externo porque de esta manera se tiene una referencia clara para estudiar los diferentes procesos del sistema. La información así obtenida se utiliza en la detección de actividad nerviosa normal/anormal, localización de problemas referidos a un segmento del canal nervioso, caracterización de la severidad de un problema, intensidad de una respuesta emocional, etc.

Refiriéndonos al tema de la emoción desde este marco de referencias, Salazar (2011) menciona que la emoción es la combinación de un proceso evaluador mental, simple o complejo, con determinadas respuestas a dicho proceso, la mayoría de naturaleza fisiológica, que producen un estado corporal emocional, pero también hacia el cerebro mismo, el cual produce cambios mentales adicionales. Por su parte, Ruetti, Mustaca y Bentosela (2008) argumentan que las emociones están íntimamente vinculadas con la memoria. Clásicamente se considera que el contenido emocional de los eventos influye sobre el recuerdo posterior. Al utilizar resonancia magnética funcional encontraron que la codificación de las imágenes emocionales correlacionaba con el incremento de la activación de la amígdala. Del mismo modo, sus resultados exhibieron una alta correlación entre la activación en la amígdala y del lóbulo temporal medial para las imágenes emocionales, más que para las neutras. Estos datos sugieren que la codificación de material emotivo es diferente y más intensa que la de material neutro, probablemente por la alta modulación de la amígdala sobre el lóbulo temporal medial.

Siguiendo ese planteamiento, podemos mencionar que las emociones funcionan como un sistema de filtro, seleccionando sus hechos por su significado emocional, para posteriormente ser procesados por la memoria de manera más prolongada (Rodríguez, Schafe, \& LeDoux, 2004, citados en Ruetti, et al., 2008). Pese a los significativos aportes brindados por las neurociencias hay que tener en cuenta la apreciación de Salazar (2011) que menciona que las operaciones fisiológicas que se denominan "mente" derivan de un conjunto estructural funcional y no solamente del cerebro. Los fenómenos mentales solo pueden comprenderse cabalmente en el contexto de la interacción de un organismo con su ambiente.

\section{El proceso de ventas}

Teniendo en cuenta esta serie de habilidades constituidas dentro del constructo de inteligencia emocional y las emociones que se tienen en cuenta, solo queda preguntar: ¿Qué papel juega la inteligencia emocional dentro del proceso de ventas? y icómo el percibir emociones de manera adecuada puede mejorar el rendimiento en las ventas?

El proceso de ventas es de carácter psicosocial con etapas definidas y formas específicas de comportamiento esperado entre las partes que 
desarrollan el intercambio (Biggart \& Delbridge, 2004; Argyle, 1992, citado en Javela \& Botero, 2008). Autores tales como Plouffe et al. (2013) organizan el proceso de ventas en cinco fases: rasgos y predisposiciones individuales del vendedor, las conductas de ventas, conductas relacionadas con la venta asociadas a "aguas-abajo" dentro del nivel del trato, resultados asociados y resultados de rendimiento. Estas etapas pueden ser definidas como:

1. Rasgos y predisposiciones individuales del vendedor: rasgos examinados con frecuencia en la literatura de las ventas incluyen la competitividad (Brown et al., 1997; Plouffe, Sridharan, \& Barclay, 2010) y la autoeficacia (Wang \& Netemeyer, 2002).

- Autoeficacia: representa la confianza de una persona en su capacidad para realizar tareas específicas, en especial, las que son nuevas o con las que no se está familiarizado (Krishnan, Netemeyer, \& Boles, 2002; Verbeke, Belschak, \& Bagozzi, 2004).

- Rasgo de competitividad: representa el disfrute de una persona de la competencia interpersonal y el deseo de ganar y ser mejor que otros (Spence \& Helmreich, 1983).

2. Conductas de ventas: los vendedores que tienen una fuerte capacidad de percibir las diferencias situacionales y ajustar sus interacciones con los clientes en los distintos entornos de ventas lograrán un mayor éxito. Para ajustarse a los diferentes escenarios se hace necesario que el vendedor presente:

- Adaptación en las ventas: las conductas de ventas se producen dentro y a través de las interacciones de ventas, los vendedores deben adaptar su enfoque durante estos momentos, en este sentido, vendedores altamente auto-eficaces perciben mayor libertad para ajustar su comportamiento a las demandas de la interacción de ventas (Ahearne et al., 2005).

- Orientación al cliente: se trata de establecer una relación con base en lo que necesita comprar y no trata de convencerlo de cosas que podría no necesitar (Saxe \& Weitz, 1982).
3. Conductas relacionadas con las ventas asociadas a "aguas-abajo" dentro del nivel del trato: Investigadores como Deighton y Narayandas (2004) afirman que la parte final del proceso de ventas se produce en términos de profundas negociaciones entre el comprador y el vendedor antes de la decisión final de compra. Como tal, sugieren la necesidad de examinar el comportamiento del vendedor más "aguas-abajo" en el ciclo de ventas, es decir mucho más allá del punto de "adaptación" al cliente o de la adaptación de la presentación de uno (Spiro \& Weitz, 1990).

4. Resultados asociados: Son los resultados que se presentan después de esta negociación final en materia de opinión subjetiva, es decir si tanto el vendedor como el cliente se encuentran satisfechos y están dispuestos a entablar una relación provechosa a largo plazo (Plouffe et al., 2013).

5. Resultados de rendimiento: Es la manera en que se operacionalizan los resultados. Si bien hay varias opciones de medición de nivel de ventas para operacionalizar el rendimiento de trabajos anteriores, se ha demostrado que las medidas subjetivas de rendimiento (es decir "autoinformadas") y las objetivas, poseen fortalezas y debilidades relativas (Bommer, Johnson, Rich, Podsakoff, \& MacKenzie, 1995; Rich, Bommer, MacKenzie, Podsakoff, \& Johnson, 1999; Plouffe, Hulland, \& Wachner, 2009)

Debido a que la venta es un proceso psicosocial es importante tener en cuenta los postulados de Mueller y Curhan (2006). Estos argumentan que el modelo acerca de la inteligencia emocional de Mayer y Salovey (1997) está específicamente desarrollado para examinar la utilidad social de este constructo, además este modelo es el único que tiene una rigurosa evidencia empírica de que la inteligencia emocional está relacionada con la calidad de las relaciones de los individuos con sus pares (Lopes, Salovey. Cote, Beers, \& Petty, 2005). 
Por su parte, Curhan, Elfenbein y Xu (2006) han introducido el constructo valor subjetivo, el cual, abarca la consecuencias psicosociales de una negociación (es decir, los sentimientos, percepciones y emociones). Específicamente consta de cuatro dimensiones: los sentimientos sobre el resultado decisivo - los términos del acuerdo-, incluyendo las percepciones subjetivas sobre si el resultado económico era deseable, equilibrado y coherente con los principios de legitimidad y precedentes; los sentimientos sobre sí mismo, incluyendo el sentirse competente y satisfecho de que uno se ha comportado adecuadamente; sentimientos acerca del proceso de negociación, los sentimientos acerca de la relación, sentirse escuchado y que lo han tratado de manera justa incluyendo impresiones positivas, la confianza y una base sólida para trabajar juntos en el futuro.

Aunque relacionado con el concepto de justicia, definido como "equidad percibida" (Greenberg \& Colquitt, 2005), el valor subjetivo abarca factores adicionales que están fuera de los límites de la construcción de la justicia; como la eficiencia del tiempo y de la autoestima. También, a diferencia de la justicia, el valor subjetivo se refiere específicamente a la resolución de disputas. De forma similar es la percepción de una persona, los pensamientos y las actitudes en lugar de la realidad objetiva de una situación que influyen en el comportamiento, incluso si el vínculo no siempre es directo o transparente (Eagley \& Chaiken, 1998). Esto implica que la comprensión de valor subjetivo podría arrojar luz sobre las motivaciones y tendencias a la acción de un negociador, así como el proceso de aprendizaje de la experiencia.

El valor subjetivo que resulta de una negociación puede retroalimentar, positiva o negativamente en los futuros resultados económicos. Las personas que aumentan el valor subjetivo de sus contrapartes pueden ser capaces de desarrollar y aprovechar los beneficios de las reputaciones más favorables (Fortgang, Lax, \& Sebenius, 2003; Croson \& Glick, 2001). Un aumento del valor subjetivo propio podría aumentar la perseverancia y la motivación para trabajar en pro de los asentamientos efectivos en entornos futuros de negociación.
A nivel de relación, la relación interpersonal desarrollada podría fomentar tanto la preocupación por la otra parte, como el intercambio de información y otras conductas de negociación críticas para el éxito de esta (Drolet \& Morris, 2000; Mannix, Tinsley, \& Bazerman, 1995; Pruitt \& Rubin, 1986). De hecho, es más probable que la relación se mantenga intacta y, todavía, se lleve a cabo la venta si los negociadores establecen bases sólidas para ella (Oliver, Balakrishnan, \& Barry, 1994).

En consecuencia, los negociadores necesitan suficiente buena voluntad para poner en práctica los términos objetivos de un contrato y abordar el llamado contrato social del cómo trabajar juntos, comunicarse y resolver conflictos en el futuro (Fortgang et al., 2003; Walton, Cutcher-Gershenfeld, \& McKersie, 1994). Por lo tanto, el mantenimiento de las buenas relaciones, que puede ser obstaculizado por la extracción de todas las posibles recompensas económicas, puede ser una estrategia efectiva en el mantenimiento de la cooperación necesaria para una mayor rentabilidad en el largo plazo (Axelrod, 1984).

También es importante resaltar que los individuos capaces de manejar sus emociones y expresar ciertas de ellas en el trabajo, presentan menor cantidad de emociones negativas y facilitan las positivas (Bermúdez, Álvarez y Sánchez, 2003; Ciarrochi, Chan y Caputi, 2000, citados en Contreras, Barbosa, \& Espinoza, 2009; Gopalan, Culbestron, \& Leiva, 2012); son personas que pueden percibir, entender y regular no solo sus estados emocionales, sino que también aprecian, entienden y tratan los sentimientos y emociones ajenas (Mayer \& Salovey, 1997). La inteligencia emocional está relacionada directamente con emociones positivas tales como la satisfacción con la vida (Extremera \& Fernández-Berrocal, 2005, citados en Contreras et al., 2009). Por otra parte, la identificación de las emociones propias así como también las ajenas, su entendimiento y respuesta eficiente acerca de la información que ellas proveen sobre nosotros mismos y sobre el ambiente, pueden promover el bienestar personal (Fernández-Berro-cal, Extremera y Palomera, 2008, citados en Contreras et al., 2009). 
Otro constructo que debería ser tenido en cuenta por los vendedores es el de la empatía, definido por Ioannidou y Konstantikaki (2008) y Duffy (2007) como la habilidad de ver el mundo a través de los ojos de alguien más o ponerse en sus zapatos, lo que simplemente implica el desarrollo de la habilidad de imaginar lo que la otra persona está pensando y sintiendo en una situación determinada. Este es un intento de entender al otro, de vivir y sentir de la misma forma.

Un aporte interesante lo hace Humphrey (2013) quien asegura que la empatía también puede ayudar a los empresarios a interactuar mejor con sus clientes. Los clientes pueden percibir a un pequeño empresario que tiene alta empatía como más preocupado por sus necesidades y satisfacciones. En contraste, propietarios de pequeñas empresas con baja empatía pueden ser percibidos como preocupados únicamente por la fabricación de un beneficio. Líderes empáticos pueden ser más conscientes de los deseos, necesidades y preferencias de sus clientes $y$, por lo tanto, poseer una ventaja en la oferta de productos y servicios que los clientes desean.

Al mencionar al rendimiento dentro del proceso de ventas (Sitser et al., 2013) se destaca la etapa de las conductas referentes a las ventas y sus dos componentes: la adaptación y la orientación al cliente. La interacción entre comprador y vendedor es única, porque los vendedores tienen la flexibilidad para modificar el mensaje de ventas de acuerdo con la situación y las reacciones de los compradores (Saxe \& Weitz, 1982). Como tal, los vendedores tienen una oportunidad única para poner en práctica presentaciones de ventas adaptados a los clientes individuales y de rápido ajuste a los mensajes, en respuesta a las reacciones de los clientes (Spiro \& Weitz, 1990).

La investigación ha indicado que los vendedores de mayor rendimiento son más capaces de adaptarse a las diferentes situaciones de ventas mediante el desarrollo de guiones distintivos para diferentes clientes y, asimismo, tener en cuenta más contingencias en diferentes situaciones de ventas relativas a los diferentes tipos de clientes (Saxe \& Weitz, 1982; Weitz, Sujan, \& Sujan, 1986). Los vendedores con menor rendimiento tienden a hablar demasiado y en momentos inadecuados (Boorom, Goolsby, \& Ramsey, 1998), tendiendo a alienar a los clientes al no escuchar sus preocupaciones (Ingram, Schwepker, \& Hutson, 1992). Con la mejora en estas habilidades interpersonales se sugiere que afectarían sustancialmente de manera positiva el rendimiento de ventas (Schuster \& Danes, 1986).

La venta orientada al cliente implica la comercialización a nivel tanto del vendedor individual como del comprador (Saxe \& Weitz, 1982), también se centra en la identificación y satisfacción de las necesidades de los clientes. Este enfoque requiere que los vendedores se involucren en conductas que incrementan la satisfacción del cliente y, en consecuencia, evitar comportamientos a largo plazo que conducen a la insatisfacción (Dunlap, Dotson, \& Chambers, 1988).

\section{Discusión}

Bagozzi, et al. (1999) plantean ciertas bases sobre la importancia del reconocimiento de los estados emocionales para los vendedores y Brown et al. (1997) afirman la influencia de las emociones sobre el comportamiento y el rendimiento específicamente de los profesionales en ventas. Estas dos nociones acerca de la relación entre rendimiento en procesos de ventas y emociones se vuelve un carácter central para la integración de la inteligencia emocional en la formación en ventas.

Los reconocidos estudios de Paul Ekman sobre la universalidad de las emociones y de las expresiones faciales (ver Ekman, 2003) están de acuerdo con las investigaciones de carácter pionero desarrolladas por Mayer y Salovey (1997), no cabe duda de su aporte al descubrimiento de la inteligencia emocional. Sin embargo, a partir del conocimiento recolectado sobre la personalidad, se han encontrado resultados mixtos sobre la relación entre los cinco factores del Big Five y la inteligencia emocional. Lo anterior podría deberse al carácter complejo del concepto de inteligencia emocional y las diversas metodologías en que esta puede ser evaluada. Los resultados también permiten discutir sobre la idea de que la personalidad es la realización suprema de la conducta peculiar e innata del ser humano, 
mientras que los rasgos de personalidad poseen una relación con la inteligencia emocional; sin embargo, esta sería una de las nociones a precisar para definir qué rasgos están ligados y a qué nivel.

El aporte de Sitser et al. (2013) propuso que la optimización en las ventas está relacionada con una personalidad altruista, emocionalmente estable, agradable, consciente, extrovertida e intelectualmente abierta, desde su bienestar, satisfacción con la vida y autoestima. El estudio de la personalidad podría dar una mejor perspectiva a la selección y formación de profesionales en ventas. Las consecuencias prácticas de este estudio subrayan la necesidad de fomentar la educación de la inteligencia emocional como un medio para lograr que la persona construya una red social adecuada que facilite a su vez su adaptación psicosocial (Pennebaker, 1997, citado por Extremera \& Fernández-Berrocal, 2004). Esta idea implica que la inteligencia emocional debería incluirse en los futuros programas de mejora y entrenamiento de las habilidades sociales en el ámbito educativo.

Las neurociencias aportan la relación entre memoria y emoción, mostrando de esta forma las bases para un estudio de carácter más holístico sobre la experiencia de ventas y para el desarrollo de procesos de neuromarketing (Salazar, 2011) utilizando metodologías de carácter más objetivo. La postura de este artículo es de carácter psicosocial, sin embargo el aporte de las neurociencias no es dejado de lado y se toma en cuenta para aumentar las bases teóricas y metodológicas del estudio de la inteligencia emocional y del proceso de ventas.

\section{Conclusiones de los revisores}

Varias características definen la orientación al cliente en las ventas, incluyendo que el vendedor evalúe las necesidades de los clientes, evite el engaño, evite la alta presión durante la venta, describa productos y servicios de manera adecuada, igualmente que ayude a los clientes a tomar decisiones de compra satisfactorias (Thomas, Soutar, \& Ryan, 2001). Cada una de estas características requiere que el vendedor examine con precisión cada una de las seis emociones básicas Ekman (1999) que el comprador exhibe a menudo sutilmente. Los vendedores pueden satisfacer las necesidades del cliente cuando están en mejores condiciones para evaluar el estado emocional del mismo (Kernbach \& Schutte, 2003). Estos resultados, demuestran que es menester apreciar las habilidades incluidas dentro del constructo de inteligencia emocional las cuales comprenden el percibir, utilizar, comprender y manejar como un aspecto importante dentro del proceso de ventas. Esto se evidencia de forma particular si estas habilidades son utilizadas con el fin no solo de concretar una venta, sino también con el fin de crear una relación de confianza entre comprador y vendedor, dicha relación puede afectar la probabilidad de lograr más ventas en el futuro (Kernbach \& Schutte, 2003).

Igualmente, se hace necesaria una capacitación en materia de percepción emocional para los vendedores, siempre y cuando esté acompañada de las etapas correspondientes al proceso de ventas, especialmente, tanto las conductas de ventas como las orientaciones y la adaptación al cliente, ya que sin esta habilidad el buen desempeño en las ventas se puede ver mermado (Acar, 2010). Los resultados de la investigación de Kidwell, Macfarland y Ávila (2007) afirman que los vendedores que tienen bajos niveles de percepción emocional no solo reducen el uso eficaz de los comportamientos relacionados con la venta, sino de hecho, pueden afectar negativamente las relaciones con los clientes. Cuando los vendedores son incapaces de leer las emociones, los clientes pueden sentirse como si sus necesidades no se estuvieran cumpliendo y los vendedores no se preocuparan por estas.

Tales transacciones (es decir, la venta) pueden crear sentimientos contraproducentes de desconfianza e insatisfacción que pueden ser costosos para la empresa. Es importante proporcionar a los gerentes de venta una manera de identificar a las personas con bajas habilidades para percibir emociones y hacer arreglos para que sean capacitados para ser perceptivos, más eficaces, de aquí viene la importancia de crear una herramienta interactiva en forma de software para ayudar a fomentar estas habilidades y por tanto, presentar un mejor rendimiento a través de su uso, de la adaptación y de técnicas de ventas orientadas al cliente. 


\section{Contribución de los autores}

La reflexión e integración de las teorías revisadas, en particular las referentes a la inteligencia emocional y la teoría de las emociones básicas, nos lleva a mirar una aplicabilidad que en los actuales momentos en Colombia puede llegar a ser de relevancia. Estos aportes conceptuales pueden mejorar las condiciones de vida de las personas dedicadas a las ventas porque, al mejorar las condiciones del trabajo se mejora el bienestar de los trabajadores (Polo-Vargas, Palacio Sañudo, De Castro Correa, Mebarak Chams, \& VelillaGuardela, 2013). Esto implica que la construcción de un software para el mejoramiento de las ventas podría ayudar, además del beneficio económico, a incrementar la autoeficacia que, según Salanova y Schaufeli (2009), suele ser una variable a destacar en los procesos de la organización. Para finalizar, la construcción y adecuación del software podría permitir en un futuro integrar variables culturales a la hora de mejorar la eficacia de las personas en las ventas.

\section{Referencias}

Acar, F. P. (2010). Analyzing the effects of diversity perceptions and shared leadership on emotional conflict: a dynamic approach. International Journal of Human Resource Management, 21(10), 1733-1753.

Ahearne, M., Jelinek, R., \& Jones, E. (2007). Examining the Effect of Salesperson Service Behavior in a Competitive Context, Journal of the Academy of Marketing Science, 35, 603-616.

Ambler, T., Braeutigam, S., Stins, J., Rose, S., \& Swithenby, S. (2004). Salience and choice: neural correlates of shopping decisions. Psychology and Marketing, 21(4), 247-261.

Ather, M., \& Bano, M. (2013). Emotional Intelligence and Personality Traits among University Teachers: Relationship and Gender Differences. International Journal of Business and Social Science, 4(17), 253-259.

Axelrod, R. (1984). The Evolution of Cooperation. New York: Basic Books.
Bagozzi, R., Gopinath, M., \& Nyer, U. (1999). The role of emotions in marketing. Journal of the Academy of Marketing Science, 27, 184-206.

Biggart, N., \& Delbridge, R. (2004). "Systems of Exchange". Academy of Management Review, 29(1), $28-49$.

Bommer, W., Johnson, J., Rich, A., Podsakoff, P., \& MacKenzie, S. (1995). On the Interchangeability of Objective and Subjective Measures of Employee Performance: A Meta-Analysis. Personnel Psychology, 48(3), 587-605.

Boorom, M., Goolsby, J., \& Ramsey, R. (1998). Relational Communication Traits and Their Effect on Adaptiveness and Sales Performance. Journal of the Academy of Marketing Science, 26, 16-30.

Briñol, P., Gandarillas, B., Horcajo, J., \& Becerra, A. (2010). Emoción y metacognición: Implicaciones para el cambio de actitud. Revista de Psicología Social, 25, 157-183.

Brown, S., Cron, W., \& Slocum, J. (1997). Effects of goal-directed emotions on salesperson volitions, behavior and performance: a longitudinal study. Journal of Marketing. 61, 39-50.

Chopra, P., \& Kanji, G. (2010). Emotional Intelligence. A catalyst for inspirational leadership and management excellence. Total quality management, 21(10), 971-1004

Clopton, S., Stoddard, J., \& Clay, J. (2002). Salesperson Characteristics Affecting Consume Complaint Responses. Journal of Consumer Behavior, 1, 124 139.

Contreras, F., Barbosa, D., \& Espinoza, J. (2009). Personalidad, inteligencia emocional y afectividad en estudiantes universitarios de áreas empresariales Implicaciones para la formación de líderes. Revista Diversitas-perspectivas en psicología, 6(1), 65-79.

Cortés, J., Barragán, C., \& Vásquez, M. (2002). Perfil de inteligencia emocional: construcción, validez y confiabilidad. Salud mental, 25(5), 50-60.

Croson, R., \& Glick, S. (2001). Reputations in Negotiations. In S. Hoch \& H. Kunreuther (Eds.), Wharton on Making Decisions. (pp. 177-186). New York: Wiley.

Curhan, J., Elfenbein, H., \& Xu, H. (2006). What do people value when they negotiate? Mapping the 
domain of subjective value in negotiation. Journal of Personality and Social Psychology, 91, 493-512.

Deighton, J., \& Narayandas, D. (2004). Commentary on 'Evolving to a New Dominant Logic for Marketing. Journal of Marketing, 68, 18-27.

Drolet, A., \& Morris, M. (2000). Rapport in conflict resolution: Accounting for how face-to-face contact fosters mutual cooperation in mixed-motive conflicts. Journal of Experimental Social Psychology, 36, 26-50.

Duffy, J. (2007). Empathy, Neutrality and Emotional Intelligence: A Balancing Act for the Emotional Einstein. Queensland University of Technology Law and Justice Journal. 186, 18(3), 44-61.

Dunlap, B., Dotson, M., \& Chambers, T. (1988). Perceptions of Real-Estate Brokers and Buyers: A SalesOrientation, Customer-Orientation Approach, Journal of Business Research, 17, 175-188.

Eagly, A. H. \& Chaiken, S. (1998). Attitude structure and function. En D. T. Gilbert, S. T. Fiske and G. Lindzey (Eds.), The Handbook of Social Psychology (4th edn., Vol. 1, pp. 269-322). New York: McGraw-Hill.

Ekman, P. (1992). Are they basic emotions? Psychological review, 99, 550-553.

Ekman, P. (1994). All emotions are basic. The nature of emotion: fundamental questions. New York: Oxford University Press.

Ekman, P. (1999). Basic emotions. Handbook of cognition and emotion. New York: Wiley.

Ekman, P. (2003). Darwin, deception, and facial expression. Annals of the New York Academy of Sciences, 1000, 205-221.

Extremera, N., \& Fernández-Berrocal, P. (2004). Inteligencia emocional, calidad de las relaciones interpersonales y empatía en estudiantes universitarios. Clínica y Salud. 15(2), 117-137.

Fortgang, R., Lax, D., \& Sebenius, J. (2003). Negotiating the spirit of the deal. Harvard Business Review, 1(9), 3-11.

García-Izquierdo, A., Ramos-Villagrasa, P., \& GarcíaIzquierdo, M. (2009). Los Big Five y el Efecto Moderador de la Resistencia en el Agotamiento Emocional. Revista de Psicología del Trabajo y de las Organizaciones, 25, 135-147.
Gardner, H. (1983). Frames if mind. The theory of multiples intelligences. Basic Books. New York.

Gopalan, N., Culbestron, S., \& Leiva, P. (2012). Explaining Emotional Labor's Relationships with Emotional Exhaustion and Life Satisfaction: Moderating Role of Perceived Autonomy. Universitas psychologica, 12, 347-356.

Greenberg, J., \& Colquitt, J. (2005). The handbook of organizational justice. Mahwah, NJ: Erlbaum.

Hassan, J., \& Mohsen, J. (2012). Management: a study of an organizational culture and the relationship between emotional intelligence and communication effectiveness. Journal of management research, 41(1), 1-14.

Humphrey, R. (2013). The Benefits of Emotional Intelligence and Empathy to Entrepreneurship. DE GRUYTER, 3(3), 287-294. http://dx.doi. org/10.1515/erj-2013-0057.

Ingram, T., Schwepker, C., \& Hutson, D. (1992).Why Salespeople Fail. Industrial Marketing Management. 21, 225-231.

Ioannidou, F., \& Konstantikaki, V. (2008). Empathy and emotional intelligence: What is it really about? International Journal of Caring Sciences, 1(3) 118-123.

Javela, L., \& Botero, M. (2008). El fenómeno del cliente leal como una relación social: un estudio con vendedores ambulantes de café en la ciudad de Ibagué. Univesitas psichologica, 8, 183-198

Kernbach, S.T., \& Schutte, N. (2003). Emotional Intelligence of service providers as a determinant of transaction-specific customer satisfaction. Australian Journal of Psychology, 55, 188-189.

Kidwell, B., McFarland, R., \& Avila, R. (2007). Perceiving emotion in the buyer-seller interchange: the moderated impact on performance. Journal of Personal Selling EZ Sales Management, 27(2), 119-132.

Krishnan, B., Netemeyer, R., \& Boles, S. (2002). SelfEfficacy, Competitiveness, and Efforts as Antecedents of Salesperson Performance. Journal of Personal Selling $\mathcal{E}$ Sales Management, 22(4), 285-295.

Lopes, P., Salovey. P., Cote, S., Beers, M., \& Petty, R. (2005). Emotion regulation abilities and the quality in social interaction. Emotion, 5, 113-118.

Mannix, E., Tinsley, C., \& Bazerman, M. (1995). Negotiating over time: Impediments to integrative solu- 
tions. Organizational Behavior $\mathcal{E}$ Human Decision Processes, 62, 241-251.

Mayer, J., \& Salovey, P. (1997). What is emotional intelligence? New York: Basic Books.

Mayer, J. D., Salovey, P., \& Caruso, D. R. (2002). "Mayer-Salovey-Caruso Emotional Intelligence Test (MSCEIT)" item booklet. Canada: MHS Publishers.

Mueller, J., \& Curhan, J. (2006). Emotional Intelligence and counterpart mood induction in a negotiation. International journal of conflict management, 17(2), $110-128$

Nawin, N., Redzuan, M., \& Hamsan, H. (2012). Inter Relationship between Emotional Intelligence and Personality Trait of Educator Leaders. International Journal of Academic Research in Business and Social Sciences, 2(5), 223-237.

Oliver, R.L., Balakrishnan, P.V., \& Barry, B. (1994) Outcome satisfaction in negotiation: a Test of expectancy disconfirmation. Organizational Behavior and Human Decision Processes, 60, 252-275.

Palmer, B., Gignac, G., Manocha, R., \& Stough, C. (2005). A psychometric evaluation of the MayerSalovey-Caruso Emotional Intelligence Test Version 2.0. Intelligence, 33, 285-305.

Perestelo-Perez, L. (2013). Standards on how to develop and report systematic reviews in psychology and health. International Journal of Clinical and Health Psychology, 13(1), 49-57.

Plouffe, C., Holmes, Y., \& Beuk, F. (2013). Testing an enhanced, Process-Based View of The sales Process. Journal of Personal Selling $\mathfrak{G}$ Sales Management, 33(2), 141-163.

Plouffe, C., Hulland, J., \& Wachner, T. (2009). Customer-Directed Selling Behaviors and Performance: A Comparison of Existing Perspectives. Journal of the Academy of Marketing Science, 37(4), 422-439.

Plouffe, C., Sridharan, S., \& Barclay, D.(2010). Exploratory Navigation and Salesperson Performance: Investigating Selected Antecedents and Boundary Conditions in High-Technology and Financial Services Contexts. Industrial Marketing Management, 39(4), 538-550.

Polo-Vargas, J. D., Palacio Sañudo, J. E., De Castro Correa, A. M., Mebarak Chams, M. R., \& Velilla Guardela, J. L. (2013). Riesgos psicosociales: la psicología organizacional positiva como propuesta de promoción, prevención e intervención. Salud Uninorte, 29(3), 561-575.

Pruitt, D. G., \& Rubin, J. Z. (1986). Social conflict: Escalation, stalemate, and settlement. New York: McGraw-Hill.

Rich, G., Bommer, W., MacKenzie, S., Podsakoff, P., \& Johnson, J. (1999). Apples and Apples or Apples and Oranges? A Meta-Analysis of Objective and Subjective Measures of Salesperson Performance. Journal of Personal Selling EF Sales Management, 19(4), 41-52.

Rodríguez, U., \& Suárez, Y. (2012). Relación entre inteligencia emocional, depresión y rendimiento académico en estudiantes de psicología. Psicogente, 15(28), 348-359.

Rodríguez, U., Amaya, A., \& Argota, A. (2011). Inteligencia emocional y ansiedad en estudiantes universitarios. Psicogente, 14(26), 310-320.

Ruetti, E., Mustaca, A., \& Bentosela, M. (2008). Memoria Emocional: Efectos De La Corticosterona Sobre Los Recuerdos. Revista Latinoamericana de Psicología, 40(3), 461-474.

Salazar, C. (2011). La neurociencia del consumidor como horizonte de investigación, conceptos y aplicaciones. Un enfoque paradigmático. Universidad $\mathcal{E}$ Empresa, 21, 143-166.

Salanova, M., Schaufeli, W.B. (2009). El engagement en el trabajo. Cuando el trabajo se convierte en pasión. Madrid: Alianza Editorial.

Salovey, P., \& Grewal, D. (2005). The science of emotional intelligence. American Psychological Society, 14(6), 330-339.

Saxe, R., \& Weitz, B. (1982). The SOCO scale: A measure of the customer orientation of salespeople. Journal of Marketing Research, 19, 343-351.

Schuster, C., \& Danes, J. (1986). Asking Questions: Some Characteristics of Successful Sales Encounters. Journal of Personal Selling $\mathcal{E}$ Sales Management, 6 (1), 17-28.

Sitser, T., van der Linden, D., \& Born, M. Ph. (2013). Predicting Sales Performance Criteria with Personality Measures: The Use of the General Factor of Personality, the Big Five and Narrow Traits, Human Performance, 26(2), 126-149, doi:10.1080/ 08959285.2013 .765877 
Spence, J., \& Helmreich, R. (1983). Achievement-related motives and behavior. In J. T. Spence (Ed.) Achievement and Achievement Motives: Psychological Approaches (pp. 7-74). San Francisco: Freeman.

Spiro, R., \& Weitz, A. (1990). Adaptive Selling: Conceptualization, Measurement, and Nomological Validity. Journal of Marketing Research, 27(1), 61-69.

Thomas, R., Soutar, G., \& Ryan, M. (2001). The Selling Orientation-Customer Orientation (S.O.C.O.) Scale: A Proposed Short Form. Journal of Personal Selling E⿱ Sales Management, 21(1), 63-69.

Trujillo, M., \& Rivas, L. (2008). Emotional intelligence model for directors of research centers in Mexico. Estudios Gerenciales, 24(106), 13-30.

Vecchiato, G.; Astolfi, L.; De Vico Fallani, F.; Toppi, J.; Aloise, F., Bez, F.,... Babiloni, F. (2011) On the Use of EEG or MEG Brain Imaging Tools in Neuromarketing Research. Computational intelligence and neuroscience, 2011, doi: 10.1155/2011/643489
Verbeke, W., Belschak, F., \& Bagozzi, R. (2004). The Adaptive Consequences of Pride in Personal Selling. Journal of the Academy of Marketing Science, 32(4), 386-402.

Walton, R., E., Cutcher-Gershenfeld, J. E., \& McKersie, R. B. (1994). Strategic negotiations: Theory of change in labor-management relations. Cambridge, MA: Harvard Business School Press.

Wang, G., \& Netemeyer, R. (2002). The Effects of Job Autonomy, Customer Demandingness, and Trait Competitiveness on Salesperson Learning, SelfEfficacy, and Performance. Journal of the Academy of Marketing Science, 30(3), 217-228.

Weitz, B., Sujan, H., \& Sujan, M. (1986). Knowledge, Motivation, and Adaptive Behavior: A Framework for Improving Selling Effectiveness. Journal of Marketing, 50, 174-191. 